\title{
Avaliação do efeito da temperatura de calcinação nas características estruturais e morfológicas de aluminas sintetizadas pelo método Pechini
}

\author{
Effect of calcination temperature on the \\ structural characteristics and morphology of \\ aluminas synthesized by the Pechini
}

Mirele Costa ${ }^{1}$, Helio Lira ${ }^{2}$, Poliana Ribeiro $^{3}$, Normanda Freitas ${ }^{4}$

\author{
${ }^{1}$ Laboratório de Síntese - LabsMAC - UFCG - CEP: 58429-900, Campina Grande, PB. \\ mirelecsilva@hotmail.com \\ ${ }^{2}$ Universidade Federal de Campina Grande, Unidade Acadêmica de Engenharia de Materiais, Avenida Aprígio Veloso, \\ 882, 58429-900. Campina Grande, Paraíba. \\ ${ }^{3}$ Universidade Federal de Campina Grande - Unidade Acadêmica de Tecnologia do Desenvolvimento. Rua Luiz Grande, \\ S/N - CEP 58540-000 - Sumé - Paraíba. \\ ${ }^{4}$ Universidade Federal da Paraíba, Departamento de Engenharia de Energias Renováveis. Centro de Engenharia de Ener- \\ gias Renováveis. Cidade Universitária, CEP: 58051-900 - João Pessoa - Paraíba. \\ helio@dema.ufcg.edu.br, normanda@dema.ufcg.edu.br, pollyana@cear.ufpb.br
}

\section{RESUMO}

O presente trabalho tem como objetivo sintetizar alumina pelo método Pechini e avaliar a influência da temperatura de calcinação nas características estruturais e morfológicas das mesmas com o intuito de direcionálas a distintas aplicações. A relação utilizada na metodologia para o ácido cítrico:cátion metálico foi de 3:1 e as temperaturas de calcinação variaram de 500 a $1200^{\circ} \mathrm{C}$ em intervalos de $100^{\circ} \mathrm{C}$. Os materiais sintetizados foram caracterizados por difração de raios X, distribuição granulométrica e microscopia eletrônica de varredura. Os resultados demonstraram que a variação da temperatura de calcinação alterou a estrutura e a morfologia da alumina. As amostras apresentaram-se amorfas abaixo da temperatura de calcinação a $800^{\circ} \mathrm{C}$, a fase alumina surgiu após a calcinação a $900^{\circ} \mathrm{C}$ e a fase alfa alumina após $1100^{\circ} \mathrm{C}$. $\mathrm{O}$ aumento da temperatura na calcinação diminuiu o diâmetro médio dos aglomerados de 17,79 para 13,01 $\mu \mathrm{m}$ para as amostras calcinadas a 500 e $1200^{\circ} \mathrm{C}$, respectivamente. Quanto à morfologia, a mudança na temperatura de calcinação não alterou de forma significativa o material sintetizado. As partículas estão distribuídas aleatoriamente ao longo das amostras apresentando geometria com aparência de placas em diferentes dimensões depositadas sobre notáveis aglomerados. As características geradas pelas aluminas possibilitam sua aplicação em membranas, catálise, implantes odontológicos, compósitos, etc.

Palavras-chave: Alumina, temperatura de calcinação, método Pechini.

\section{ABSTRACT}

The present work aims to synthesize alumina by Pechini method and evaluate the influence of calcination temperature on structural and morphological characteristics of the same in order to direct them to different applications. The methodology used in relation to citric acid : metal cation was 3:1 and calcination temperatures ranging $500-1200^{\circ} \mathrm{C}$ in steps of $100^{\circ} \mathrm{C}$. The synthesized materials were characterized by X-ray diffraction, particle size distribution and scanning electron microscopy. The results showed that the variation of the calcination temperature altered the structure and morphology of the alumina. The samples had amorphous below the calcination temperature to $800^{\circ} \mathrm{C}$, the alumina phase appeared after calcination at $900^{\circ} \mathrm{C}$ and the alpha alumina phase after $1100^{\circ} \mathrm{C}$. The increase in calcination temperature decreased the average diameter of 
the agglomerates of 17.79 to $13.01 \mu \mathrm{m}$ for samples calcined at 500 and $1200^{\circ} \mathrm{C}$, respectively. The morphology, the change in calcination temperature did not change significantly the synthesized material. The particles are randomly distributed throughout the samples presenting geometry appearance of plaques in different sizes deposited on notable clusters. The features generated by the aluminas allow the application of alumina membranes, catalysis, dental implants, composites, etc.

Keywords: Alumina, temperature of calcination, Pechini method.

\section{INTRODUÇÃO}

Quando comparado a outros óxidos, a alumina tem atraído o interesse intenso por apresentar partícula fina, elevada área de superfície e boa atividade catalítica. A alumina é, dentro das cerâmicas avançadas, a mais utilizada por oferecer bom desempenho em termos de resistência mecânica, à corrosão e a alta dureza a um bom custo/benefício. Oferece uma boa combinação de propriedades mecânicas e elétricas, o que favorece a sua utilização em uma grande gama de aplicações. Além disso, a alumina pode ser produzida em diferentes percentuais de pureza e/ou formar outros compósitos cerâmicos, aprimorando suas propriedades [1]].

A alumina está dentre os novos materiais utilizados para alcançar propriedades de maior tenacidade à fratura com menor fragilidade, boa biocompatibilidade, alta dureza e resistência ao desgaste, o que representa destaque entre outros materiais, já que apresentam essa combinação de propriedades [2] .

Dentre diversas aplicações a que se destina a obtenção desses materiais, podem ser citados, por exemplo, fabricação de compósitos []], revestimento em substratos em vidros de silício e de aço inoxidável [4]], implantes como ortoplastias totais de quadril [్], em biotecnologia [ㅁ] , aplicações com espectros de ultravioleta [7], dentre outras.

A alumina é o principal componente da bauxita, que industrialmente, é purificada por meio do processo Bayer para obtenção do óxido de alumínio. Apesar de ser frequentemente descrita como o minério de alumínio, a bauxita não é uma espécie mineral propriamente dita, mas um material heterogêneo formado de uma mistura de hidróxidos de alumínio hidratados $\left(\left[\mathrm{AlO}_{\mathrm{x}}(\mathrm{OH})_{3-2 \mathrm{x}}\right], 0<\mathrm{x}<1\right)$ contendo impurezas [8]

$\mathrm{O}$ processo industrial para obtenção de alumina apresenta desvantagens como: problemas de corrosão e possibilidade de outros hidróxidos de alumina se dissolver durante o processo, dificuldade no controle de pureza, além de ser um processo dividido em várias etapas. Por essas razões junto à busca de melhores propriedades estruturais e morfológicas, pesquisadores têm voltado suas atenções para o uso de métodos químicos que permitem a obtenção do óxido de alumínio em laboratório, esses métodos têm apresentado resultados eficazes para obtenção de diversos materiais. Dentre os métodos não convencionais que a literatura vêm apresentando para síntese de aluminas podem ser citados: o método por reação de combustão [9], a síntese hidrotermal [10], a termólise [2] e o método Pechini [11].

Na escolha do método a ser utilizado na síntese de nanopartículas, alguns parâmetros devem ser considerados mais importantes, sendo aqueles que se direcionam a um melhor controle de área específica, menor tamanho de partículas e baixo grau de aglomeração [12].

Entre os métodos químicos, o método Pechini tem apresentado resultados satisfatórios no que diz respeito à síntese de óxido de alumínio [11]. Segundo Kakihana [13] o processo Pechini oferece muitas vantagens sobre as reações no estado sólido convencional, tais como menores temperaturas e tempo de processamento, maior homogeneidade e pureza e maior capacidade para fabricar filmes finos feitos com pós de partículas submicrométricas. Entre as vantagens desse método está a possibilidade de preparação de complexos de boa homogeneidade a nível molecular e controle estequiométrico. As temperaturas requeridas são mais baixas do que no método convencional de mistura de óxidos e permite a obtenção de pós com alta pureza [14], é um método direto e preciso com mistura de multicomponentes em escala molecular. Também se destaca em relação aos outros métodos de síntese química, pois garante uma composição química reprodutível, com granulometria controlada e com estrutura cristalina estável [12].

Atualmente, em muitos casos, toma-se por base a patente de Pechini [15] e utilizam-se variações dela. É possível variar a relação ácido cítrico:cátion metálico ou ainda as quantidades entre ácido cítrico/etilenoglicol. É possível incorporar os mais diversos cátions metálicos na rede polimérica, os quais acabam distribuídos homogeneamente. Porém, em seus estudos, Pechini [15] reportou que a melhor relação de ácido cítrico/etilenoglicol é de (40/60)\% em massa. Várias pesquisas têm sido voltadas a avaliar como essas relações têm alterado, na maioria das vezes de forma significativa, as propriedades dos materiais obtidos e gerado uma melhor qualidade na aplicação a que se destina o material produzido [12].

O objetivo desse trabalho é realizar a avaliação do efeito da temperatura de calcinação sobre as características estruturais e morfológicas de aluminas sintetizadas pelo método Pechini, mantendo a mesma rela- 
ção, 3:1 de ácido cítrico:cátion metálico, para a relação de ácido cítrico:etilenoglicol de (40/60)\% em massa.

\section{MATERIAIS E MÉTODOS}

Os materiais utilizados para síntese da alumina pelo método Pechini foram: ácido cítrico monohidratado $\mathrm{C}_{6} \mathrm{H}_{8} \mathrm{O}_{7} \cdot \mathrm{H}_{2} \mathrm{O}$ (NUCLEAR), nitrato de alumínio - $\mathrm{Al}\left(\mathrm{NO}_{3}\right)_{3} .9 \mathrm{H}_{2} \mathrm{O}$ (VETEC) e etilenoglicol $-\mathrm{C}_{2} \mathrm{H}_{8} \mathrm{O}_{2}$ (VETEC). Os cálculos para as quantidades dos reagentes utilizados encontram-se em anexo.

A síntese da alumina pelo método Pechini foi realizada de acordo com a relação molar ácido cítrico:cátion metálico na proporção de 3:1. O etilenoglicol foi adicionado a uma razão de 40/60 (\% em massa) em relação ao ácido cítrico de acordo com a metodologia proposta por Pechini [15].

No início do processo foi efetuada a diluição do ácido cítrico em $400 \mathrm{ml}$ de água destilada dentro de um Becker posto sobre uma placa aquecedora com agitação constante a $70^{\circ} \mathrm{C}$. Após a homogeneização completa da solução foi adicionado o nitrato de alumínio. A solução permaneceu na mesma temperatura $\left(70^{\circ} \mathrm{C}\right) \mathrm{e}$ agitação durante $24 \mathrm{~h}$ para favorecer a etapa de esterificação. Passadas as $24 \mathrm{~h}$ foi adicionado o etilenoglicol e a temperatura da placa foi elevada para $110^{\circ} \mathrm{C}$, onde foi obtida uma resina polimérica. Ainda dentro do Becker o material foi transferido para um forno mufla a $400^{\circ} \mathrm{C} / 1 \mathrm{~h}$ a uma taxa de aquecimento de $10^{\circ} \mathrm{C} / \mathrm{min}$ para etapa de pirólise.

A etapa de pirólise foi realizada conforme os seguintes patamares: temperatura inicial de $25^{\circ} \mathrm{C}$ até $400^{\circ} \mathrm{C}$, a uma taxa de aquecimento de $10^{\circ} \mathrm{C} / \mathrm{min}$ e tempo de permanência de $60 \mathrm{~min}$.

Uma amostra do material pirolisado foi para análise termogravimétrica em um analisador térmico, modelo RB - 3000 - 20, fabricado pela empresa BP Engenharia, com taxa de aquecimento de $12,5^{\circ} \mathrm{C} / \mathrm{min}$, em atmosfera de nitrogênio, usando um cadinho de platina e faixa de temperatura da ambiente $\left(25^{\circ} \mathrm{C}\right)$ até a temperatura máxima de $1000^{\circ} \mathrm{C}$.

Os pós obtidos da pirólise foram desaglomerados em almofariz e passados em peneira malha \#325 e posteriormente levados a etapa de calcinação para formação da fase cristalina nas seguintes temperaturas: $500,600,700,800,900,1000,1100$ e $1200^{\circ} \mathrm{C}$. A etapa de calcinação foi realizada em forno elétrico da marca JUNG seguindo os seguintes patamares: temperatura inicial de $25^{\circ} \mathrm{C}$ até a temperatura máxima de calcinação $\left(500,600,700,800,900,1000,1100\right.$ e $\left.1200^{\circ} \mathrm{C}\right)$, a uma taxa de aquecimento de $10^{\circ} \mathrm{C} / \mathrm{min}$ e tempo de permanência de $60 \mathrm{~min}$.

As amostras calcinadas foram caracterizadas por análise granulométrica em um analisador de tamanho de partículas por difração a laser (granulômetro), marca Cilas, modelo $1064 \mathrm{LD}$, com faixa analítica de 0,04 a $500 \mu \mathrm{m}$ e emissores de laser secundários para uma melhor precisão na faixa do sub-mícron, por difração de raios X em um difratômetro de raios X modelo XRD-6000 da Shimadzu com radiação k $\alpha$ do cobre e varredura de 10 a $80^{\circ}$. A cristalinidade foi determinada a partir da razão entre a área integrada do pico referente à fase cristalina e a área referente à fração amorfa. $\mathrm{O}$ tamanho médio de cristalito foi calculado a partir da linha de alargamento de raios $\mathrm{X}\left(\mathrm{d}_{311}\right)$ através da deconvolução da linha de difração secundária do silício policristalino (utilizado como padrão) utilizando-se a equação de Scherrer [16]. Por meio do gráfico de WilliamsonHall pode-se expressar o comportamento linear das amostras utilizando a Equação (1) de Williamson-Hall [17].

$$
\frac{\beta \times \cos \theta}{\lambda}=\frac{k}{D}+\frac{4 \varepsilon}{\lambda} \operatorname{sen} \theta
$$

onde $\beta$ é a largura a meia altura do pico (FWHM), $\lambda$ é comprimento de onda dos raios X e $\mathrm{k}$ é a constante que depende da simetria da reflexão e que em geral adota-se $\mathrm{k}=1$. O coeficiente angular e o coeficiente linear do gráfico $\beta \cos \theta /(\lambda)$ versus $\operatorname{sen} \theta$, que foi aproximado por uma reta (regressão linear: $y=b+a . x)$, nos fornecendo um valor aproximado para a microdeformação. $O$ coeficiente linear $b$ é igual a $1 / \mathrm{D}$, onde $\mathrm{D}$ é o diâmetro médio do tamanho de cristalito e o coeficiente angular a é igual a $4 \varepsilon / \lambda$ onde $\varepsilon$ é a microdeformação e $\lambda$ é o comprimento de onda dos raios X, que é igual a 1,54 A. 
As amostras foram caracterizadas também por microscopia eletrônica de varredura em um microscópio eletrônico de varredura da Shimadzu, modelo SSX-550.

Para facilitar a interpretação e apresentação dos resultados, as amostras foram nomeadas em Al.05, Al.06, Al.07, Al.08, Al.09, Al.10, Al.11 e Al.12, para as amostras calcinadas a 500, 600, 700, 800, 900, 1000, 1100 e $1200^{\circ} \mathrm{C}$, respectivamente

\section{RESULTADOS}

A Figura 1 ilustra os resultados de análise térmica das amostras sintetizadas pelo método Pechini após a etapa de pirólise a $400^{\circ} \mathrm{C} / 1 \mathrm{~h}$.

Observa-se por meio da análise térmica a ocorrência de duas etapas de perdas de massa. Uma perda inicial entre as temperaturas de 25 e $317^{\circ} \mathrm{C}$ de $0,56 \mathrm{~g}$ referente a eliminação da parte orgânica ainda presente no material e uma segunda etapa entre as temperaturas de 317 e $1000^{\circ} \mathrm{C}$ onde ocorreu a maior perda de material atingindo um valor de $1,79 \mathrm{~g}$ equivalente a decomposição da matéria orgânica.

No intervalo de temperatura entre 317 e $1000^{\circ} \mathrm{C}$ ocorre uma perda de massa mais acentuada entre 317 e $650^{\circ} \mathrm{C}$, esse resultado auxilia nos casos em que o material produzido será levado para alguma etapa de sinterização, como por exemplo, em aplicações para membranas cerâmicas, onde para as faixas de temperaturas de maiores perdas de massa, a taxa de aquecimento deve ser baixa para evitar trincas e/ou rachaduras no material a ser produzido. No total a perda de massa do material sintetizado foi de $2,35 \mathrm{~g}$.

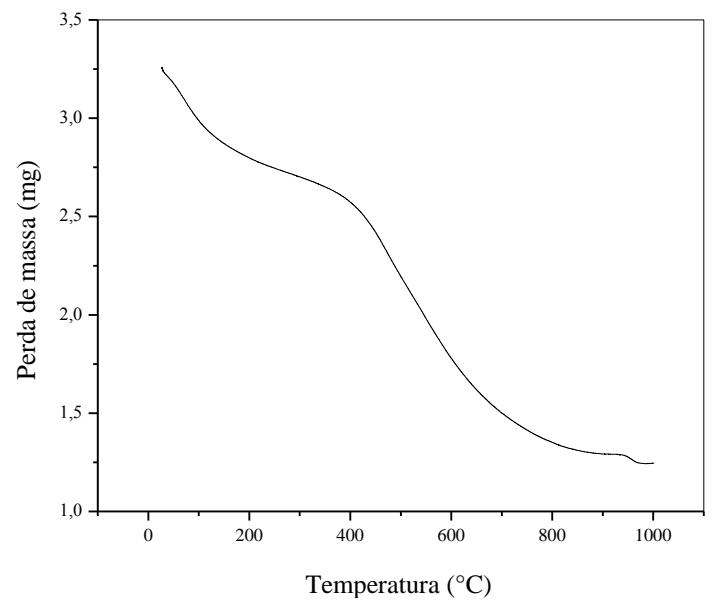

Figura 1: Análise térmica da amostra sintetizada na proporção 3:1 de ácido cítrico:cátion metálico, antes da etapa de calcinação.

Percebe-se, portanto, uma alta perda de massa durante o aquecimento, isso ocorre pelo fato de que o material pirolisado se apresenta altamente desordenado estruturalmente e rico em matéria orgânica. Com a elevação da temperatura tem-se consequentemente a ruptura da cadeia polimérica, ocasionando transformações estruturais no material e eliminação da matéria orgânica.

Tais resultados são compatíveis com os resultados reportados na literatura quando utilizado o método Pechini para síntese de alumina, dentre tais, o trabalho feito por Zaki et al. [18].

A Figura 2 ilustra os difratogramas dos pós obtidos pelo método Pechini calcinados em diferentes temperaturas.

A fim de facilitar a interpretação dos difratogramas, a Tabela 1 apresenta os dados das fases presentes em cada amostra, a ficha padrão de cada uma, tamanho médio de cristalito, cristalinidade e microdeformação média.

TABELA 1: Características estruturais das amostras sintetizadas pelo método Pechini calcinadas em diferentes temperaturas. 


\begin{tabular}{c|c|c|c|c|c}
\hline AMOSTRAS & $\begin{array}{c}\text { FASE } \\
\text { PRESENTE }\end{array}$ & FICHA PADRÃO & $\begin{array}{c}\text { TAMANHO MEDIO DE } \\
\text { CRISTALITO }(\mathbf{n m})\end{array}$ & $\begin{array}{c}\text { CRISTALINIDADE } \\
(\%)\end{array}$ & $\begin{array}{c}\text { MICRODEFORMAÇÃO } \\
\text { MÉDIA (\%) }\end{array}$ \\
\hline $\mathrm{Al} .05$ & $*$ & $*$ & $*$ & $*$ & $*$ \\
\hline $\mathrm{Al} .06$ & $*$ & $*$ & $*$ & $*$ & $*$ \\
\hline $\mathrm{Al} .07$ & $*$ & $*$ & $*$ & $*$ & $*$ \\
\hline $\mathrm{Al} .08$ & $*$ & $*$ & 06,3 & 32,8 & 0,01045 \\
\hline $\mathrm{Al} .09$ & Alumina & $10-0425$ & 06,8 & 33,4 & 0,00969 \\
\hline $\mathrm{Al} .10$ & Alumina & $4-0877$ & 52,5 & 91,2 & 0,00186 \\
\hline $\mathrm{Al} .11$ & $\alpha$-alumina & $10-0173$ & 61,3 & 97,2 & 0,00196 \\
\hline $\mathrm{Al} .12$ & $\alpha$-alumina & $10-0173$ & &
\end{tabular}

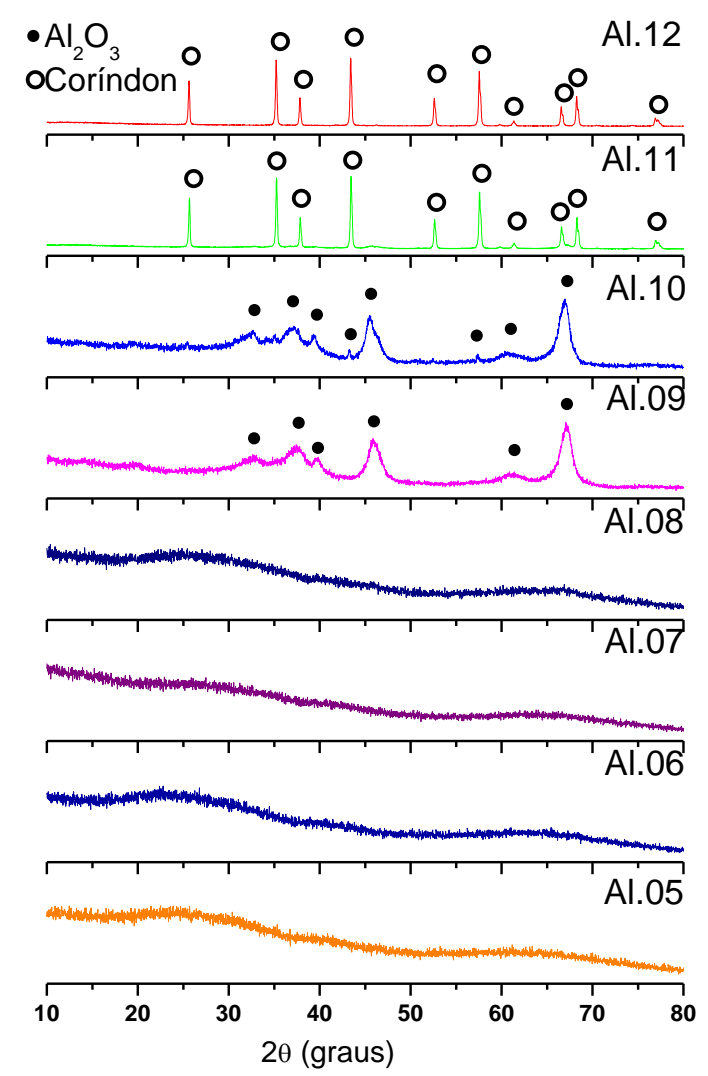

Figura 2: Difratogramas obtidos a partir de difração de raios $\mathrm{X}$ das amostras sintetizadas pelo método Pechini, calcinadas em diferentes temperaturas.

Pode-se observar por meio dos difratogramas que entre as temperaturas de calcinação de 500 a $800^{\circ} \mathrm{C}$ as amostras obtidas são amorfas, ou seja, ausente de fases cristalinas. A fase da alumina surgiu somente após a calcinação na temperatura de $900^{\circ} \mathrm{C}$ e a fase $\alpha$-alumina, óxido mais significativo devido às suas propriedades mecânicas superiores e estabilidade a temperaturas elevadas [19], após a temperatura de calcinação de $1100^{\circ} \mathrm{C}$. A fase referente a alumina calcinada a $900^{\circ} \mathrm{C}$ foi identificada através da ficha padrão JCPDF 100425 e a alumina calcinada a $1000^{\circ} \mathrm{C}$ por meio da ficha padrão JCPDF 4-0877. A partir da temperatura de calcinação de $1100^{\circ} \mathrm{C}$ a amostra sintetizada atingiu a fase $\alpha$-alumina através da ficha padrão JCPDF 10-0173.

Quanto à avaliação do tamanho de cristalito, verifica-se que o aumento da temperatura de calcinação gerou um aumento nos valores médios dos mesmos, o que, segundo Ribeiro et al. [20], é devido ao coalescimento de pequenos cristais. Os valores para os tamanhos de cristalito apresentados pelas aluminas calcinadas nessas duas temperaturas não variou de forma significativa, alcançando $6,27 \mathrm{~nm}$ para a alumina calcinada a $900^{\circ} \mathrm{C}$ e $6,78 \mathrm{~nm}$ para a alumina calcinada a $1000^{\circ} \mathrm{C}$. A elevação da temperatura de 1100 para $1200^{\circ} \mathrm{C}$ elevou o valor do tamanho de cristalito de 52,51 para $61,28 \mathrm{~nm}$, respectivamente.

Comparando esses resultados a outros reportados na literatura tem-se que Cordeiro et al. [9] ao sintetizar alumina por reação de combustão em uma resistência elétrica atingiu um tamanho de cristalito de $57 \mathrm{~nm}$ 
e Freitas et al. [21] para atingir tamanho cristalito de $61 \mathrm{~nm}$ sintetizou a $\alpha$-alumina por reação de combustão sendo necessária a realização de uma etapa de moagem durante $30 \mathrm{~min}$.

No que se refere a cristalinidade das amostras, o aumento da temperatura de calcinação também acarretou em um maior percentual de cristalinidade chegando ao valor máximo de $97,2 \%$ para a $\alpha$-alumina calcinada a $1200^{\circ} \mathrm{C}$. A maior energia proporcionada pela maior temperatura de calcinação possibilita uma maior eliminação de matéria orgânica que possivelmente ainda permanecia na amostra.

Em se tratando da microdeformação, esse parâmetro indica a deformação da célula unitária do material obtido, portanto quanto maior a cristalinidade menor esta microdeformação. À medida que o tamanho de cristal diminui, ocorre um mzaior alargamento do pico e diminuição da sua altura, o que se aproxima mais de um material com característica amorfa, portanto a microdeformação aumenta. Dessa forma os menores valores de microdeformação foram apresentados pelas amostras que apresentaram maior cristalinidade, nesse caso, as amostras Al.11 e Al.12.

A Figura 3 ilustra os resultados da distribuição granulométrica das aluminas obtidas pelo método Pechini calcinadas em diferentes temperaturas.
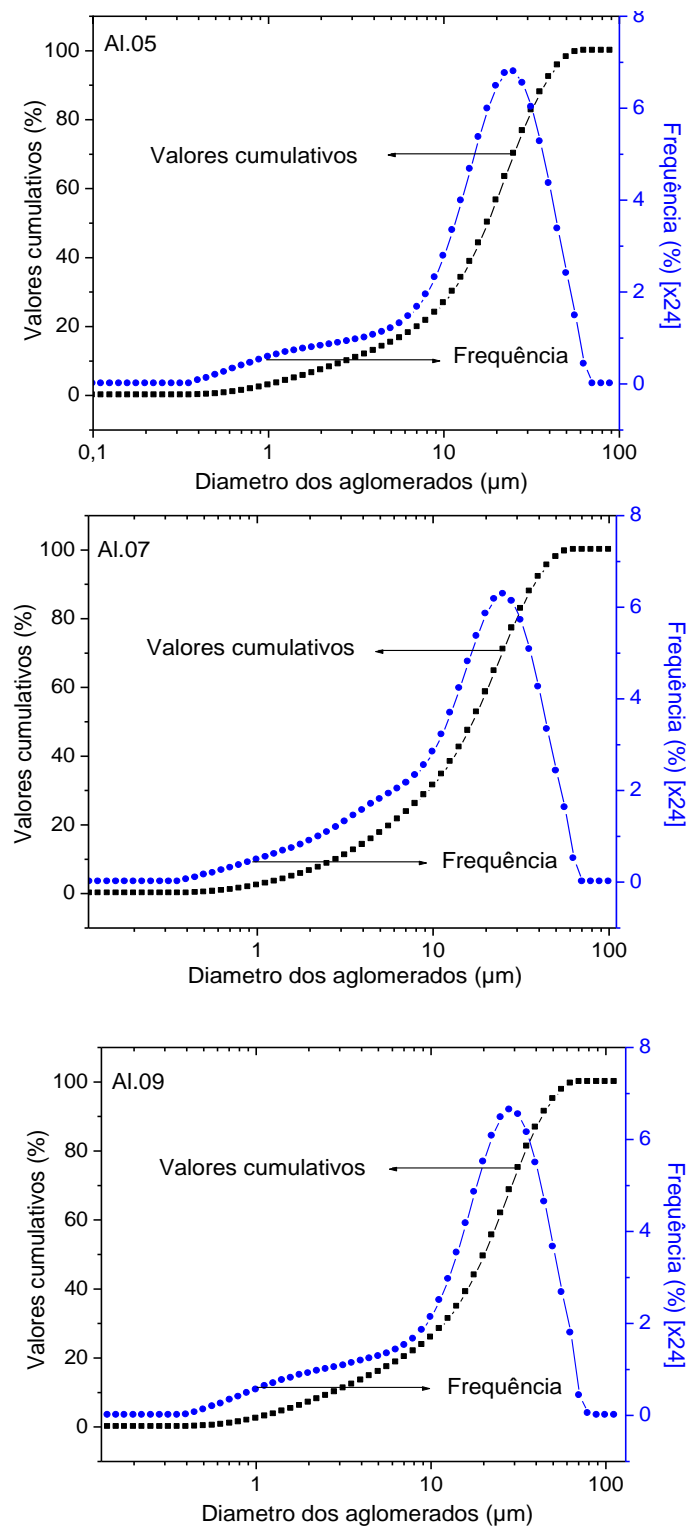
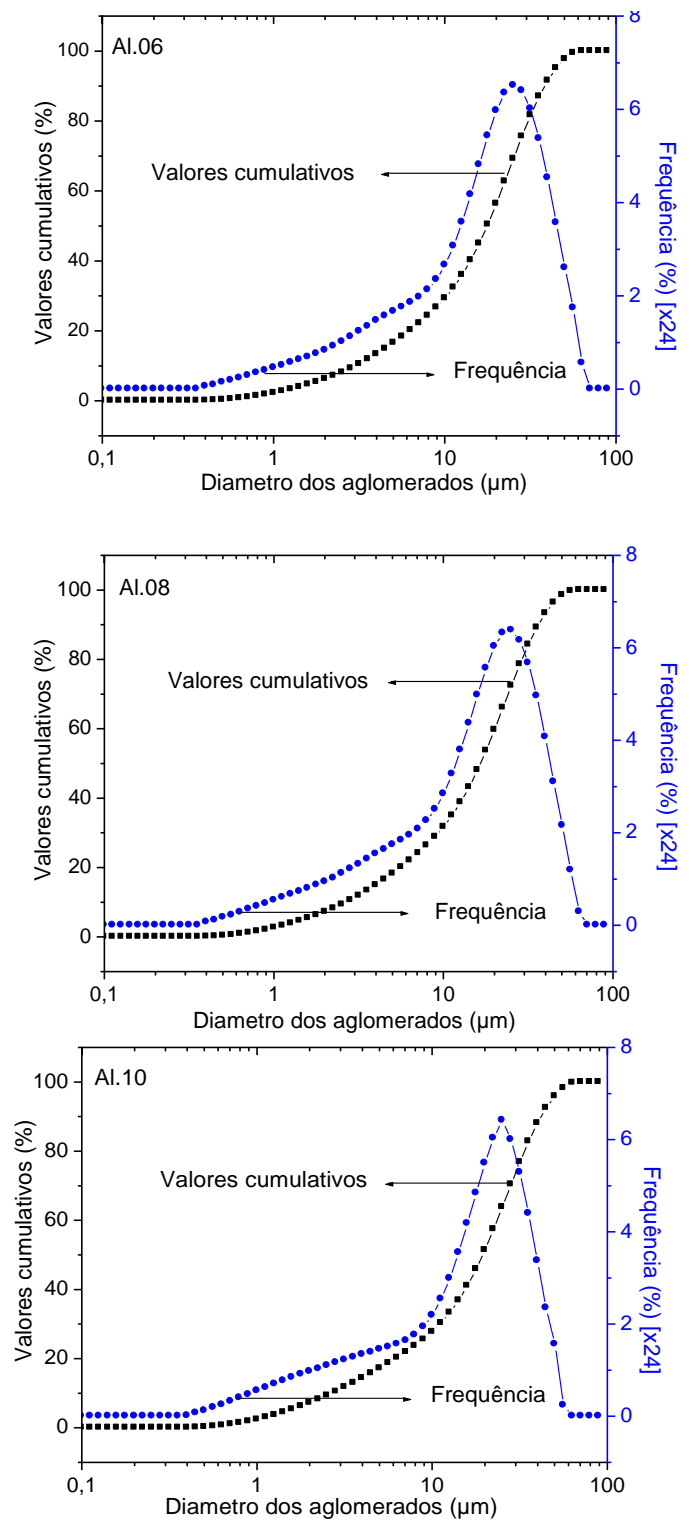

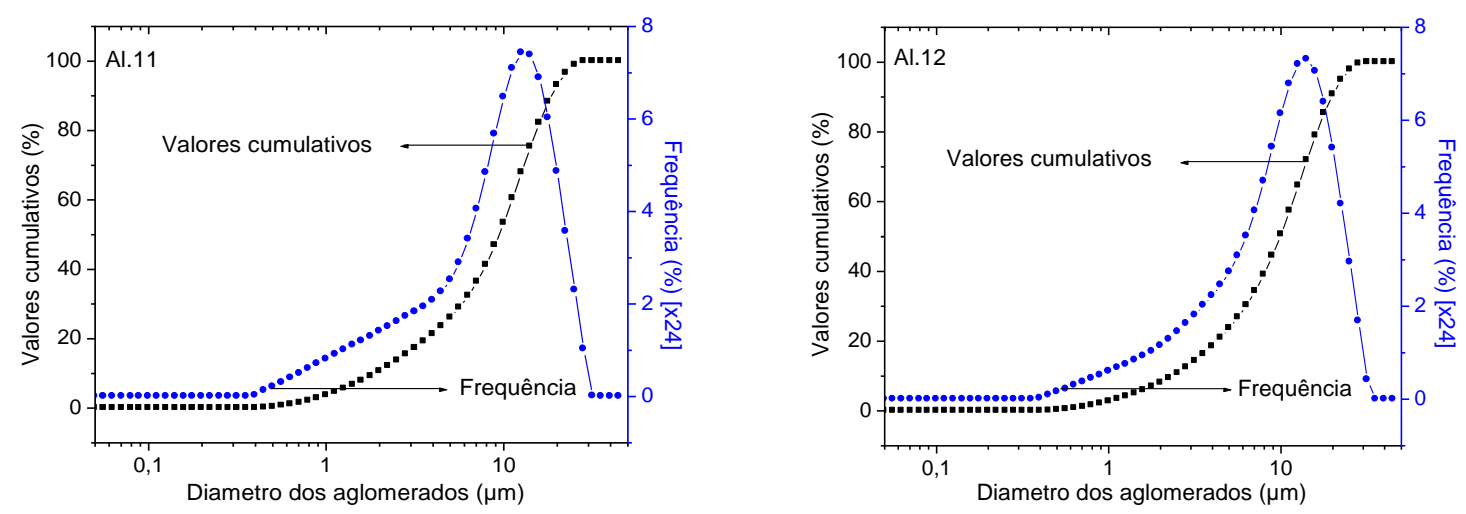

Figura 3. Distribuição granulométrica das aluminas sintetizadas pelo método Pechini calcinadas em diferentes temperaturas.

Baseado nos gráficos ilustrados na Figura 3 percebe-se que as amostras calcinadas nas diferentes temperaturas apresentam uma faixa larga quanto à distribuição de diâmetro dos aglomerados ilustrando um modelo monomodal. Ambas as curvas, tanto a de valores acumulados quanto às de freqüência foram semelhantes para todas as amostras. Comparando as curvas de frequiência verifica-se que apesar de bandas similares para todas as amostras, a partir da amostra Al.09, onde foram identificadas a fases cristalinas referentes às aluminas, essas bandas tornam-se mais simétricas.

A Figura 4 exibe o comportamento do diâmetro mediano dos aglomerados das aluminas em função do aumento da temperatura de calcinação.

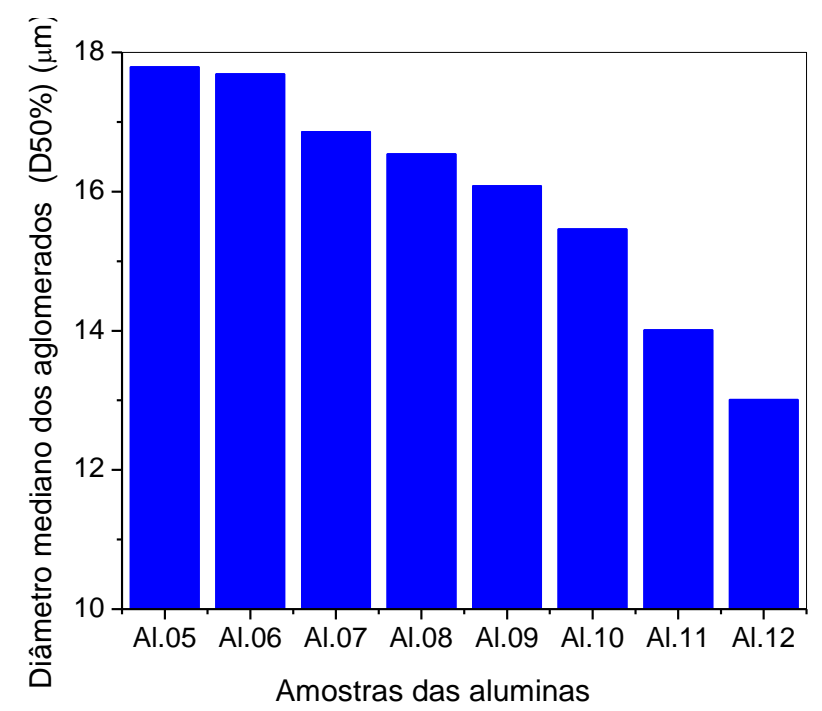

Figura 4: Comportamento do diâmetro mediano dos aglomerados em função da temperatura de calcinação das amostras sintetizadas pelo método Pechini.

Com base na Figura 4 fica mais fácil a visualização e a interpretação do comportamento do diâmetro mediano dos aglomerados das amostras em função da temperatura de calcinação. À medida que a temperatura de calcinação foi elevada o tamanho mediano do diâmetro dos aglomerados diminuiu atingindo os valores de 17,$79 ; 17,69 ; 16,86 ; 16,54 ; 16,08 ; 15,46 ; 14,10$ e $13,01 \mu \mathrm{m}$ para as amostras Al.05, Al.06, Al.07, Al.08, Al.09, Al.10, Al.11 e Al.12, respectivamente. Essa variação foi mais acentuada quando elevada a temperatura de calcinação de 1000 para $1100^{\circ} \mathrm{C}$, transição de temperatura na qual surgiu a fase $\alpha$-alumina. $\mathrm{O}$ maior valor foi alcançado pela amostra Al.05 e o menor valor pela amostra Al.12.

A distribuição do tamanho de aglomerados junto a dependência da pureza química, densidade, estrutura do pó e distribuição do tamanho de poros no material são responsáveis pelas principais propriedades da alumina, dentre elas: a alta estabilidade térmica, baixa condutividade térmica, alta resistividade elétrica e alta resistência química. 
A Figura 5 ilustra as micrografias obtidas por microscopia eletrônica de varredura das amostras sintetizadas pelo método Pechini calcinadas em diferentes temperaturas.
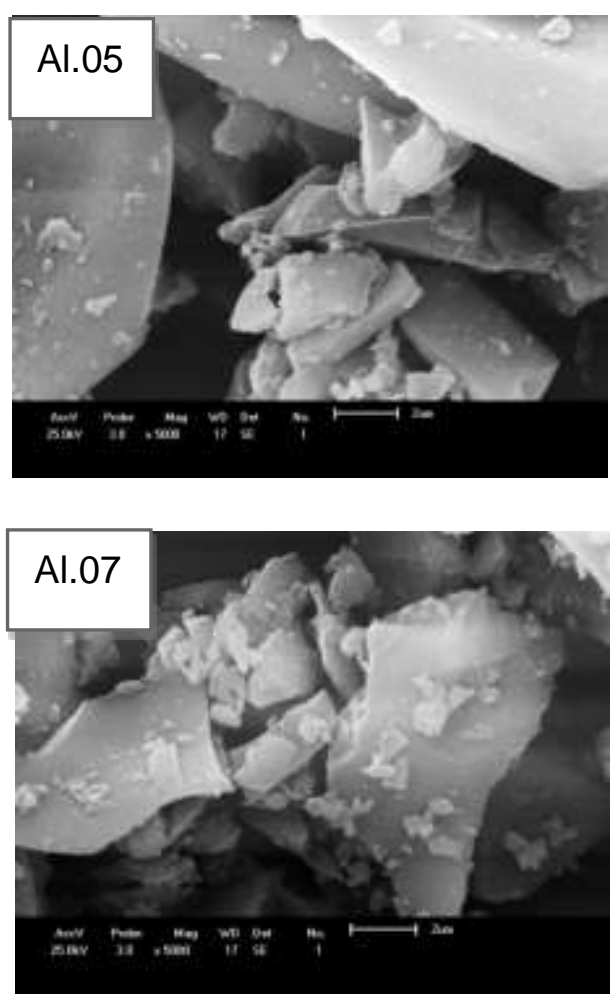
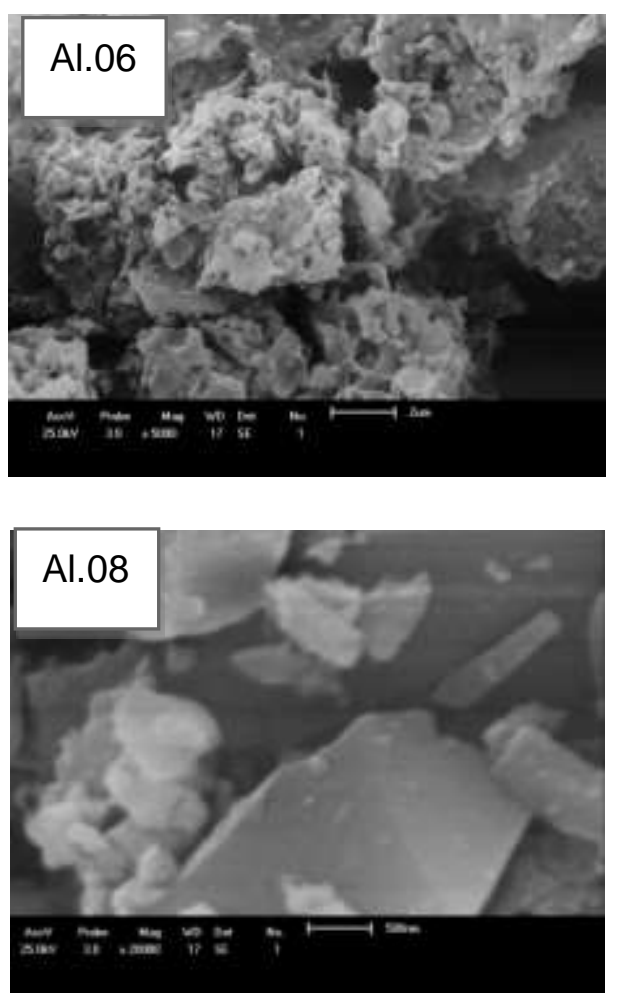

\section{Al.09}

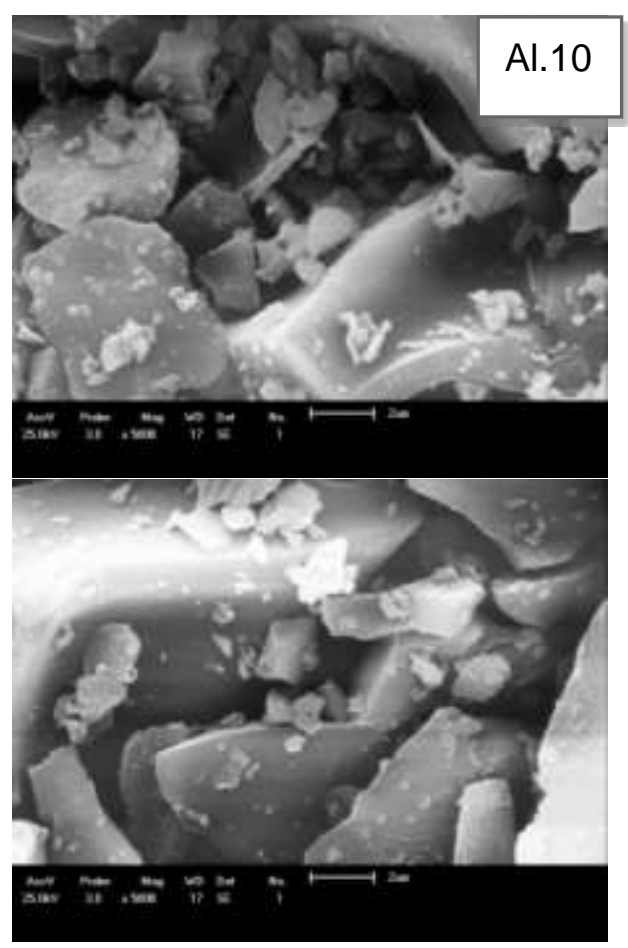



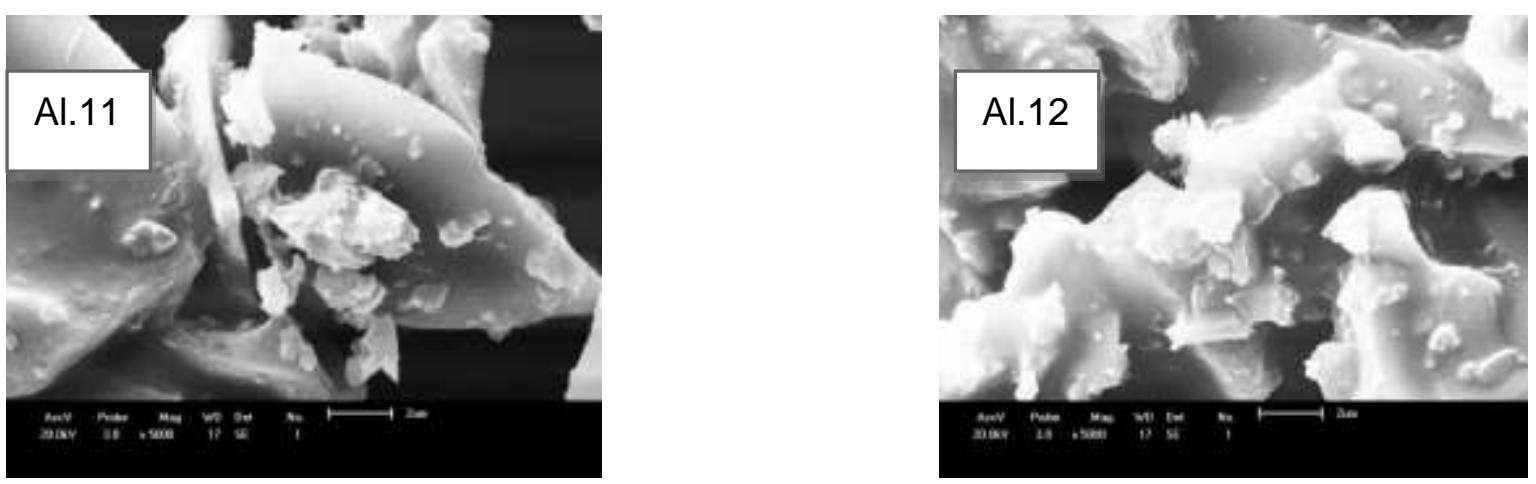

Figura 5: Micrografias das aluminas sintetizadas pelo método Pechini calcinadas em diferentes temperaturas.

No que se refere à morfologia das amostras verifica-se por meio das micrografias que a alteração da temperatura de calcinação não influenciou de forma significativa à morfologia das aluminas como ocorrido na estrutura. No geral as partículas estão distribuídas aleatoriamente ao longo das amostras apresentando geometria com aparência de placas em dimensões diversas depositadas sobre notáveis aglomerados, que, segundo a literatura [22] é uma característica típica do processo Pechini, com aspecto denso, de difícil desaglomeração, distribuídos de formas e tamanhos variados.

A mesma morfologia das partículas, com aspecto de placas também foi observada por Bitencourt et al. [23] quando sintetizou alumina pelo método Pechini e a calcinou a $1100^{\circ} \mathrm{C}$. Segundo Tanaka et al. [24], apresentando-se sob diferentes graus de hidratação, as aluminas podem ser obtidas com diferentes propriedades morfológicas e texturais, dependendo das condições empregadas durante a síntese do hidróxido precursor e do tratamento térmico de transformação deste hidróxido em uma alumina de transição.

Percebe-se também que o aumento da temperatura de calcinação acarretou em um maior grau de sinterização das partículas causado pela alta taxa de aquecimento que favorece o processo sinterização por difusão de contornos e como resultado da intensa combustão de compostos orgânicos usados no processo de síntese da resina ocorrida durante a etapa de pirólise.

A amostra Al.12 proporciona uma visualização clara da maior sinterização devido a maior temperatura de calcinação utilizada, nesse caso, $1200^{\circ} \mathrm{C}$.

\section{DISCUSSÃO}

No que se refere à síntese de partículas de aluminas, devido às peculiaridades que esses materiais apresentam, a literatura tem ilustrado trabalhos que analisam sua estrutura e morfologia e relacionam os diferentes métodos de obtenção com as suas qualidades para possíveis aplicações. As diferentes metodologias utilizadas influenciam no diâmetro das partículas e na transição de fases da alumina. O estudo realizado no presente trabalho possibilita e minimiza o planejamento experimental para o interesse de obtenção de aluminas com variadas estruturas e morfologias, atingidas apenas com a variação da temperatura de calcinação das mesmas, tomando com base a metodologia ilustrada, auxiliando no direcionamento para uma determinada aplicação.

O mesmo estudo da variação da temperatura de calcinação no método Pechini foi avaliada por Costa et al. [14] para síntese de óxido de zinco ZnO. Os autores verificaram que o aumento da temperatura de calcinação de $500^{\circ} \mathrm{C}$ para $700^{\circ} \mathrm{C}$ foi suficiente para acarretar alterações nas estruturas dos materiais obtidos, ocasionando a diminuição no alargamento dos picos de difração e aumento do tamanho de partícula, bem como uma redução da área superficial e consequente aumento do tamanho das partículas, além de uma maior aglomeração do material. Amaral, D. [25] também variou a temperatura de calcinação do composto $\mathrm{YBa}_{2} \mathrm{Cu}_{3} \mathrm{O}_{7}$ em 400, 700 e $900^{\circ} \mathrm{C}$ sintetizado pelo método Pechini e concluiu que essa variação também é suficiente para que haja aumento na cristalinidade do material com o aumento da temperatura. Com a variação estudada o autor pode controlar as fases apresentadas pelo material. Rida el al. [26] também fez a análise da variação da temperatura de calcinação para $\mathrm{LaNiO}_{3}$ verificando alterações nas características do material bem como em diferentes comportamentos para aplicação na área de catálise.

\section{CONCLUSÕES}

Com base nos resultados reportados na presente pesquisa conclui-se que a variação na temperatura durante a etapa de calcinação de aluminas sintetizadas pelo método Pechini é suficiente para causar alteração na estrutura e morfologia das mesmas, possibilitando dessa forma que esses materiais possam ser direcionados a di- 
versos setores referentes a suas aplicações, dentre tais podem ser citados, membranas cerâmicas, área de catálise, ou implantes odontológicos. Os resultados apresentados auxiliam e minimizam metodologias para pesquisadores que buscam determinadas características de aluminas designadas a uma determinada aplicação.

\section{AGRADECIMENTOS}

Os autores deste trabalho agradecem a CAPES pelo apoio financeiro.

\section{BIBLIOGRAFIA}

[1] MEKASUWANDUMRONG, O., TANTICHUWET, P., CHAISUK, C., et al., "Impact of concentration and $\mathrm{Si}$ doping on the properties and phase transformation behavior of nanocrystalline alumina prepared via solvothermal synthesis", Materials Chemistry and Physics, v. 107, n. 2-3, pp. 208-214, Feb. 2008.

[2] HE, H-X., LIU, Y., "A novel method to synthesis mesoporous alumina with visible emission by thermolysis of Al-based coordination polymer", Materials Letters, v. 76, n. 1, pp. 59-61, Mar. 2012.

[3] INBARAJ, S. R., FRANCIS, R. M., JAYA, N. V. A., "Processing and properties of sol gel derived alumina-carbon nano tube composites", Ceramics International, v. 38, n. 5, pp. 4065-4074, Jul. 2012.

[4] KHANNA, A., BHAT, D. G., "Nanocrystalline gamma alumina coatings by inverted cylindrical magnetron sputtering", Surface and Coatings Technology, v. 201, n. 1-2, pp.168-173, Set. 2006.

[5] ROUSSEAU, M.-A., MOUEL, S. L., GOUTALLIER, D., et al., "Résultats à long terme du couple de frottement alumine/alumine sur 98 prothèses de hanche cimentées", Revue de Chirurgie orthopédique de l'Réparatrice appareil Moteur, v. 90, n. 8, pp. 741-748, Dez. 2004.

[6] INGHAM, C. J., MAAT, Jurjen Ter., VOS, WILLEM M. de., Where bio meets nano: the many uses for nanoporous aluminum oxide in biotechnology, biotechnology advances, 12 aug. 2011.

[7] STENZEL, O., GÄBLER, D., WILBRANDT, S., et al., "Plasma ion assisted deposition of aluminium oxide and aluminium oxifluoride layers for applications in the ultraviolet spectral range", Optical Materials, v. 33, n. 11, pp. 1681-1687, Set. 2011.

[8] CONSTANTINO, V. R. L., ARAKI, KOITI., SILVA, D. O., et al.,"Preparação de compostos de alumínio a partir da bauxita: considerações sobre alguns aspectos envolvidos em um experimento didático", Química Nova, v. 25, n. 3, pp. 490-498, Mai. 2002.

[9] CORDEIRO, V. V., FREITAS, N. L., VIANA, K. M. S., et al.,"Influence of the external heating type in the morphological and structural characteristics of alumina powder prepared by combustion reaction", Materials Science Forum ,v. 660, n. 61, pp. 58-62, Out. 2010.

[10] JI, G., LI, M., LI, G., et al., "Hydrothermal synthesis of hierarchical micron flower-like $\gamma$-AlOOH and $\gamma$ $\mathrm{Al}_{2} \mathrm{O}_{3}$ superstructures from oil shale ash", Powder Technology, v. 215-216, n.1, pp. 54-58, Jan. 2012.

[11] HERNÁNDEZ, M. T., GONZÁLEZ, M., "Synthesis of resins as alpha-alumina precursors by the Pechini method using microwave and infrared heating", Journal of the European Ceramic Society, v. 22, n. 16, pp. 2861-2868, Dez. 2002.

[12] CARREÑO, N. L. V., LEITE, E. R., SANTOS, L. P. S., et al., "Síntese, caracterização e estudo das propriedades catalíticas e magnéticas de nanopartículas de $\mathrm{Ni}$ dispersas em matriz mesoporosa de $\mathrm{SiO}_{2}$ ", Química Nova, v. 25, n. 6, pp. 935-942, Ago. 2002.

[13] KAKIHANA, M. "Invited Review - "Sol-Gel" Preparation of High Temperature Superconducting Oxides", Journal of Sol-Gel Science and Technology, n. 6, pp. 7- 55, Jan. 1996.

[14] COSTA, A. C. F. M., RAMALHO, M. A. F., NEIVA, L. S., et al., "Avaliação do tamanho da partícula do ZnO obtido pelo método Pechini", Revista Eletrônica de Materiais e Processos, v. 2, n. 3, pp.14-19, Out. 2007.

[15] PECHINI, M. P. "Metedology of preparing lead and alkaline: earth, litanates and niobates and coating". Method using the same to for a capacitor. Us Patent 3.330.697, n. 3,330, 697, Jul. 1967.

[16] KLUNG, H.; ALEXANDER, L. X-ray diffraction procedures, New York: Wiley, 1962.

[17] WILLIAMSON, G. K., HALL, W. H., "X-ray line broadening from filed aluminum and wolfram", Acta Metallurgica, v. 1, p. 22-31, 1953. 
[18]ZAKI, T., KABEL, K. I., ASSAN, H. H. "Preparation of high pure $\alpha-\mathrm{Al}_{2} \mathrm{O}_{3}$ nanoparticles at low temperatures using Pechini method", Ceramics International. v. 38, n. 3, pp. 2021-2026, 2012.

[19]XU, L., WEI, S., ZHANG, D., et al., Fine structure and interface characteristic of $\alpha-\mathrm{Al}_{2} \mathrm{O}_{3}$ in molybdenum alloy, International Journal of Refractory Metals and Hard Materials, v. 41, pp. 483-488, 2013.

[20]RIBEIRO, M. A., NEIVA, L. S., MAIA, D. F., et al., "Síntese do $\mathrm{TiO}_{2}$ dopado com $\mathrm{Zr}$ por meio do método Pechini: Avaliação dos efeitos da temperatura de calcinação", Revista Eletrônica de Materiais e Processos, v. 7, n. 2, pp. 111-116, Mar. 2012.

[21]FREITAS, N. L., FAGURY NETO, E., LIRA, H. L., et al., "Combustion Synthesis of alfa- $\mathrm{Al}_{2} \mathrm{O}_{3}$ Powders". Materials Science Forum, v. 530, n. 3, pp. 631-636, Feb. 2006.

[22] MENDES, A. C., MAIA, L.J.Q., PARIS, E.C., et al., Solvent effect on the optimization of $1.54 \mu \mathrm{m}$ emission in Er-doped $\mathrm{Y}_{2} \mathrm{O}_{3}-\mathrm{Al}_{2} \mathrm{O}_{3}-\mathrm{SiO}_{2}$ powders synthesized by a modified Pechini method, Current Applied Physics, v. 13, n. 8, pp. 1558-1565, 2013.

[23] BITENCOURT, J. F. S., VENTIERI, A., GONÇALVES, K. A., et al., "A comparison between neodymium doped alumina samples obtained by Pechini and sol-gel methods using thermo-stimulated luminescence and SEM", Journal of Non-Crystalline Solids, v. 356, n. 52-54, pp. 2956-2959, Dez. 2010.

[24] TANAKA, L. B. H., IZARIO FILHO, J., MONTEIRO, W. R., et al., "Síntese e caracterização de aluminas para aplicação como suporte de catalisador". INPE e Print. v. 2, n. 1, pp. 1-6, Jul. 2004.

[25] AMRAL, D., "Otimização das condições de síntese do método Pechini para preparação de amostras cerâmicas de $\mathrm{YBa}_{2} \mathrm{Cu}_{3} \mathrm{O}_{7}-\delta ”$, Dissertação de M.Sc, UEP, Bauru, São Paulo,SP, Brasil, 2007.

[26] RIDA, K., PEÑA, M. A., SASTRE, E., et al., "Effect of calcination temperature on structural properties and catalytic activity in oxidation reactions of $\mathrm{LaNiO}_{3}$ perovskite prepared by Pechini method", Journal of Rare Earths, v. 30, n. 3, pp. 210-216, mar. 2012.

\section{ANEXO}

Os cálculos a seguir mostram como foram determinadas as quantidades dos reagentes empregados na síntese para obtenção dos pós de alumina.

- Para as diferentes relações molares entre ácido cítrico/cátion metálico e baseado na estequiometria calculada para obter $10 \mathrm{~g}$ de $\mathrm{Al}_{2} \mathrm{O}_{3}$, os cálculos seguiram da seguinte forma:

Considerando uma concentração de 1 mol de $\mathrm{Al}_{2} \mathrm{O}_{3}$, tem-se:

$$
\begin{aligned}
& 1 \mathrm{~mol} \mathrm{de} \mathrm{Al}_{2} \mathrm{O}_{3} \text {------------ } 102 \mathrm{~g} \\
& \mathrm{X} \text { mol --- } 10 \mathrm{~g} \\
& \mathrm{X}=\text { 0,098039 mols de óxido de alumínio }
\end{aligned}
$$

○ Dado que $1 \mathrm{~mol}$ de nitrato de alumínio $\left[\mathrm{Al}\left(\mathrm{NO}_{3}\right)_{3} \cdot 9 \mathrm{H}_{2} \mathrm{O}\right.$ ] equivale a $375,14 \mathrm{~g} / \mathrm{mol}$. Para o cálculo em gramas da quantidade de nitrato de alumínio segue:

$$
1 \text { mol de nitrato de alumínio ------------- 375,14 g }
$$

0,098039 mols de nitrato de alumínio ---------- X g

\section{$\underline{X=36,778 \mathrm{~g} \text { de nitrato de alumínio }}$}

- Para o cálculo de obtenção da massa em gramas do ácido cítrico nas composições usando a relação 3:1 de ácido cítrico/cátions metálicos, tem-se:

$$
\begin{aligned}
& 3 \text { mols de ácido cítrico ------------ } 1 \mathrm{~mol} \text { de nitrato de alumínio } \\
& \text { X mols -----o-- 0,098039 mols de nitrato de alumínio } \\
& \mathrm{X}=0,29411 \text { moles de ácido cítrico }
\end{aligned}
$$

Como:

$$
\begin{aligned}
& 1 \mathrm{~mol} \text { de ácido cítrico --------------- 210,14 g } \\
& 0,29411 \text { mols de ácido cítrico ------------X g }
\end{aligned}
$$

$\underline{X=61,806 \mathrm{~g} \text { de ácido cítrico }}$ 
- A razão etilenoglicol/ácido citríco foi estabelecida em 40/60\% em massa, de acordo com a metodologia proposta por (Pechini, 1967). Para o cálculo para a obtenção da massa em gramas do etilenoglicol, temse:

$61,806 \mathrm{~g}$ de ácido cítrico $60 \%$

$$
\begin{aligned}
& \text { X g etilenoglicol ------------- } 40 \% \\
& X=41,204 \mathrm{~g} \text { de etilenoglicol }
\end{aligned}
$$

Dado que a densidade do etilenoglicol é $1,1132 \mathrm{~g} / \mathrm{cm}^{3}$ e que $\rho=\left(\frac{m}{V}\right)$ tem-se:

$$
\begin{gathered}
\rho=\left(\frac{m}{V}\right) \\
1,1132 \mathrm{~g} / \mathrm{cm}^{3}=\left(\frac{41,204 g}{V}\right)
\end{gathered}
$$

$\mathrm{V}=37,014 \mathrm{~cm}^{3}$ de etilenoglicol 